\title{
Structure-Function-Relationship in Thylakoids Influenced by the Pyridazinone BAS 13-338 (SAN 9785)
}

\author{
Josef A. Graf, Reto J. Strasser, and Ulrich Kull \\ Biologisches Institut, Universität Stuttgart, Ulmer StraBe 227, D-7000 Stuttgart 60 , \\ Bundesrepublik Deutschland
}

Z. Naturforsch. 42c, 808-812 (1987); received October 31, 1986

Pyridazinone, Fatty Acid Desaturation. Energy Distribution, Structure-Function Relationship

The pyridazinone BAS 13-338 (SAN 9785) inhibits the desaturation sequence leading to polyunsaturated fatty acids, mainly of glycolipids. Parallel to the inhibition of fatty acid desaturation in the presence of the pyridazinone, changes in energy-distribution parameters have been observed. These data indicate that the amount of polyunsaturated fatty acids in glycolipids is strongly correlated with excitation. trapping, grouping and dissipation, but not with spillover. Functional changes in energy distribution induced by BAS 13-338 are interpreted as a consequence of structural changes in the lipid matrix, which may imply a structure-function relationship between pigment protein complexes and the surrounding lipid environment in thylakoids.

\section{Introduction}

First steps of light transforming reactions in photosynthesis take place within heterogeneously distributed pigment protein complexes in thylakoid membranes [1] and include light absorption-, exciton transfer- and charge separation-processes. It has been proposed that the pattern of exciton transfer i.e. the energy distribution behaviour influences the photosynthetic efficiency [2]. In order to coordinate energy distribution, a high degree of membrane organization might be necessary. The membrane organization seems to be characterized not only by the arrangement of pigment protein complexes but also by the interactions with the lipid environment. Therefore, the thylakoid specific lipid complement which contains a high amount of polyunsaturated fatty acids may reflect the functional role of the lipid matrix in these membranes. Several attempts have been made to gain insight into the functional interactions between acyl lipids and LHC, reaction centers

\footnotetext{
Abbreviations: PS I, II, photosystem I. II; LHC, light harvesting complex; RC I, II, reaction center I, II; MGDG, monogalactosyldiacylglycerol; DGDG, digalactosyldiacylglycerol; SQDG, sulfoquinovosyldiacylglycerol; PG, phosphatidylglycerol; PL, other phospholipids; $F_{1(0)}, F_{1(\mathrm{v})}, F_{1(\mathrm{M})}$. initial, variable and maximal fluorescence of PS I; $F_{2(0)}$, $F_{2(v)}, F_{2(\mathrm{M})}$, initial, variable and maximal fluorescence of PS II; $V$, normalized variable fluorescence of PS II; $s$. integral of non-fluorescence; 18:3, linolenic acid; BAS 13-338 (SAN 9785), 4-chloro-5-(dimethylamino)-2phenyl-3(2H)pyridazinone.

Reprint requests to Josef A. Graf.

Verlag der Zeitschrift für Naturforschung, D-7400 Tübingen 0341-0382/87/0600-0808 \$01.30/0
}

or herbicide binding protein [3-5]. The effect of high amounts of polyunsaturated fatty acids, especially $18: 3$ in acyl lipids on structure-function relationship between lipid matrix and pigment protein complexes as well as on arrangement of these complexes can be studied by inhibiting fatty acid desaturation sequence with 4-chloro-5-(dimethylamino)2-phenyl-3(2H)pyridazinone (BAS 13-338, SAN 9785). This compound has been shown to be a potent state change inhibitor [6] and a PS II electron transport inhibitor in vivo and in vitro [7]. However, it inhibits different desaturation steps in the synthesis of polyunsaturated fatty acids $[8,9]$ leading to an increase of appressed/non-appressed regions in thyla. koids [10].

In this paper we show the effect of an altered lipid matrix induced by the pyridazinone BAS 13-338 and the functional role of polyunsaturated fatty acids in acyl lipids on energy distribution in thylakoids.

\section{Materials and Methods}

4-6 week old Petunia hybrida plants (cyanidine type) were treated with $160 \mu \mathrm{M}$ BAS $13-338$ in glass chambers under $15 \mathrm{~W} / \mathrm{m}^{2}$ light intensity, $20 \pm 2{ }^{\circ} \mathrm{C}$ growth temperature and $80 \pm 10 \%$ relative humidity for $7 \mathrm{~d}$. Low temperature fluorescence kinetic measurements of leaf discs were carried out using excitation light of $633 \mathrm{~nm}$ ( $\mathrm{NeHe}$ laser, $20 \mathrm{~W} / \mathrm{m}^{2}$ ), a multibranched fiber optic and 2 photomultipliers. Fluorescence signals measured at $695 \mathrm{~nm}$ (PS II) and at $735 \mathrm{~nm}$ (PS I) were digitized on line (IMSAI 8080 microcomputer) and stored in core memory for data 
handling. Absolute $77 \mathrm{~K}$ fluorescence emission spectra [11] measured from 600 to $800 \mathrm{~nm}$ were used to correct the $F_{(0)^{-}}$and $F_{(\mathrm{M})^{-}}$-values obtained from the kinetics. The characteristics of energy distribution, i.e. trapping, grouping, spillover and dissipation were analyzed according to the energy flux theory [12]. Lipids were extracted from purified thylakoids as described elsewhere [13] and isolated by TLC on silica gel 60 (Merck) using the acetone:benzene: water system $(91: 30: 8 \mathrm{v} / \mathrm{v} / \mathrm{v})$. Lipid bands were revealed by spraying the plates with $0.003 \%(\mathrm{w} / \mathrm{v})$ alkaline Rhodamin $6 \mathrm{G}$ solution. For quantitative fatty acid analysis, individual lipid bands were scraped from the plates and transmethylated according to [14] in the presence of methylpentadecanoate standard. After extraction, aliquots of acylmethylesters were separated by $\mathrm{GC}$ in $10^{\prime} \times 1 / 8^{\prime \prime}$ steel columns packed with $10 \%$ EGSS-X on Chromosorb W/AWDCMS (80-100 mesh) using a Varian Aerograph 2700 fitted with CDS 111 integrator. Acyl lipid bands and fatty acid methyl esters were identified by comparing them with authentic standards.

\section{Results}

Fatty acid changes during pyridazinone incubation period

Although the qualitative and quantitative acyl lipid complement as well as the chlorophyll content of thylakoids remain nearly constant (data not shown), changes in the fatty acid composition have been observed (Table I). The pyridazinone treat- ment alters the unsaturated character of thylakoids by decreasing mainly linolenic acid in favour of saturated, mono- and diunsaturated $\mathrm{C}_{16}$ and $\mathrm{C}_{18}$-fatty acids. This leads to a drastic decrease of double bond indices. These effects are more pronounced in glycolipids (MGDG, DGDG, SQDG) than in phospholipid fractions (PG, PL).

\section{Low temperature fluorescence kinetics during pyridazinone incubation period}

$77 \mathrm{~K}$ fluorescence kinetics indicate a loss in the variable fluorescence $F_{2(v)}$ and $F_{1(v)}$, a decrease in the maximal fluorescence $F_{1(\mathrm{M})}$ and an increase in the initial fluorescence $F_{2(0)}$ (Fig. 1). The ratio $F_{2(\mathrm{v})} / F_{2(\mathrm{M})}$ shown to be proportional to the trapping probability $p_{2 b}$ in an ungrouped bipartite model [15], decreases from 0.75 to 0.47 , whereas $\alpha_{N}$ which implies how much energy in PS I originates from its own light absorption remains nearly constant during a $7 \mathrm{~d}$ incubation period with BAS 13-338 (SAN 9785). Based on a grouped bipartite model, however, a decrease in the ratio $F_{2(v)} / F_{2(\mathrm{M})}$ suggests that PS II trapping and/or grouping between PS II-LHC-units may be influenced by the pyridazinone treatment. According to [12], the $F_{1}$ versus $F_{2}$ plots yield straight lines with the same slopes, indicating that spillover rate constant is not affected by an altered lipid matrix in thylakoids.

The energy flux theory predicts that the normalized variable fluorescence of PS II V versus the non-fluorescence $s$ (area growth curve) is a horizontal hyperbolic function of different curvatures at $77 \mathrm{~K}$ in the case of grouping and non-grouping. On

Table I. Effect of $160 \mu \mathrm{M}$ BAS 13-338 (SAN 9785) on the fatty acid composition of thylakoid lipids from Petunia hybrida in percentage of total fatty acid content. The values are mean \pm SD obtained from at least 4 experiments.

\begin{tabular}{llrlllllll}
\hline & & $16: 0$ & $16: 1$ & $16: 0$ & $18: 0$ & $18: 1$ & $18: 2$ & $18: 3$ & $18: 4$ \\
\hline \multirow{2}{*}{ MGDG } & Control & $4.1 \pm 0.1$ & $1.1 \pm 0.1$ & $1.0 \pm 0.1$ & $1.5 \pm 0.1$ & $1.1 \pm 0.1$ & $4.5 \pm 0.1$ & $78.9 \pm 1.0$ & $7.2 \pm 0.8$ \\
& BAS 13-338 & $10.5 \pm 0.4$ & $2.7 \pm 0.3$ & $3.0 \pm 0.2$ & $3.7 \pm 0.2$ & $3.6 \pm 0.3$ & $6.8 \pm 0.1$ & $60.5 \pm 0.7$ & $6.2 \pm 0.7$ \\
DGDG & Control & $10.3 \pm 0.7$ & $1.3 \pm 0.1$ & $1.5 \pm 0.1$ & $2.2 \pm 0.1$ & $1.5 \pm 0.1$ & $3.9 \pm 0.1$ & $74.6 \pm 1.7$ & $3.8 \pm 0.2$ \\
& BAS 13-338 & $16.2 \pm 1.0$ & $3.1 \pm 0.1$ & $3.7 \pm 0.1$ & $5.1 \pm 0.2$ & $4.3 \pm 0.1$ & $4.7 \pm 0.1$ & $57.5 \pm 1.3$ & $3.0 \pm 0.2$ \\
SQDG & Control & $31.0 \pm 0.5$ & $3.2 \pm 0.3$ & $3.7 \pm 0.8$ & $5.1 \pm 0.3$ & $3.6 \pm 0.2$ & $6.4 \pm 0.6$ & $37.5 \pm 1.9$ & $5.4 \pm 0.2$ \\
& BAS 13-338 & $40.0 \pm 0.7$ & $5.3 \pm 0.5$ & $6.4 \pm 1.4$ & $8.2 \pm 0.4$ & $7.1 \pm 0.4$ & $5.6 \pm 0.5$ & $19.0 \pm 0.9$ & $2.8 \pm 0.1$ \\
PG & Control & $30.6 \pm 2.6$ & $17.0 \pm 0.7$ & $2.9 \pm 0.1$ & $4.8 \pm 0.4$ & $4.2 \pm 0.4$ & $8.0 \pm 0.8$ & $31.0 \pm 5.4$ & $0.3 \pm 0.1$ \\
& BAS 13-338 & $31.1 \pm 2.6$ & $16.9 \pm 0.7$ & $4.6 \pm 0.1$ & $8.2 \pm 0.6$ & $7.5 \pm 0.6$ & $7.2 \pm 0.7$ & $16.3 \pm 2.9$ & $1.7 \pm 0.1$ \\
PL & Control & $26.0 \pm 1.8$ & $4.0 \pm 0.3$ & $4.4 \pm 0.7$ & $5.6 \pm 0.6$ & $4.8 \pm 0.3$ & $15.8 \pm 1.0$ & $32.5 \pm 2.5$ & $1.1 \pm 0.2$ \\
& BAS 13-338 & $28.7 \pm 2.0$ & $4.5 \pm 0.3$ & $4.8 \pm 0.8$ & $7.6 \pm 0.8$ & $6.5 \pm 0.5$ & $11.3 \pm 0.7$ & $25.1 \pm 1.9$ & $3.2 \pm 0.5$ \\
\hline
\end{tabular}




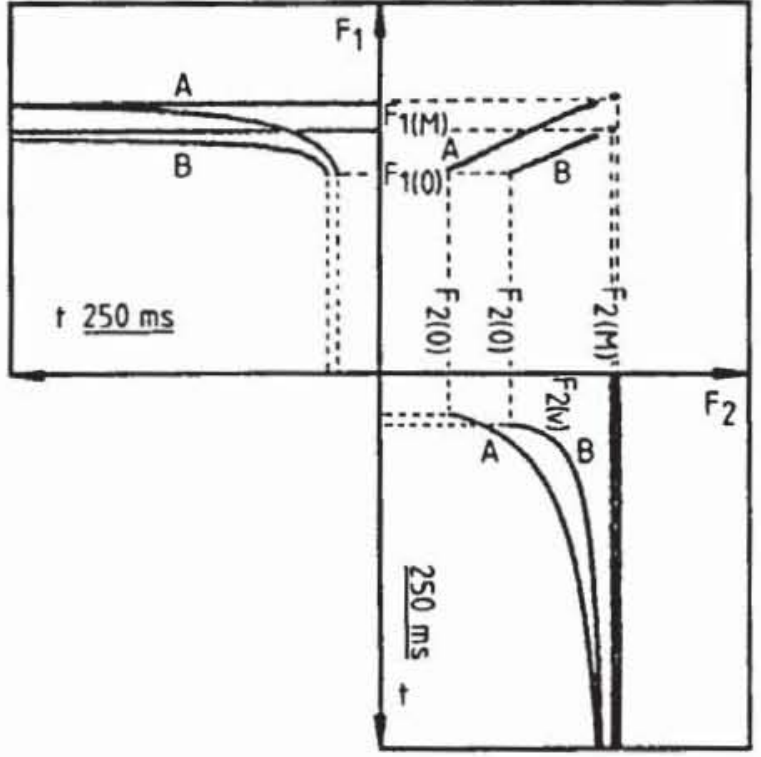

Fig. 1. Simultaneously measured fluorescence kinetics of PS I $\left(F_{1}\right)$ and PS II $\left(F_{2}\right)$ at $77 \mathrm{~K}$. (A) Control; (B) $160 \mu \mathrm{M}$ BAS 13-338 (SAN 9785) $7 \mathrm{~d}$.

this basis, the curvature of the experimental signals $F_{2(t)}$ contains the information for cooperativity of neighbouring PS II-LHC-units. Therefore, if $K$ is known, the grouping probabilities $p_{22}$ can be calculated from the intercept $V$, of the $V$ versus $V / s$ plots as shown in Fig. 2. At any time when grouping is zero

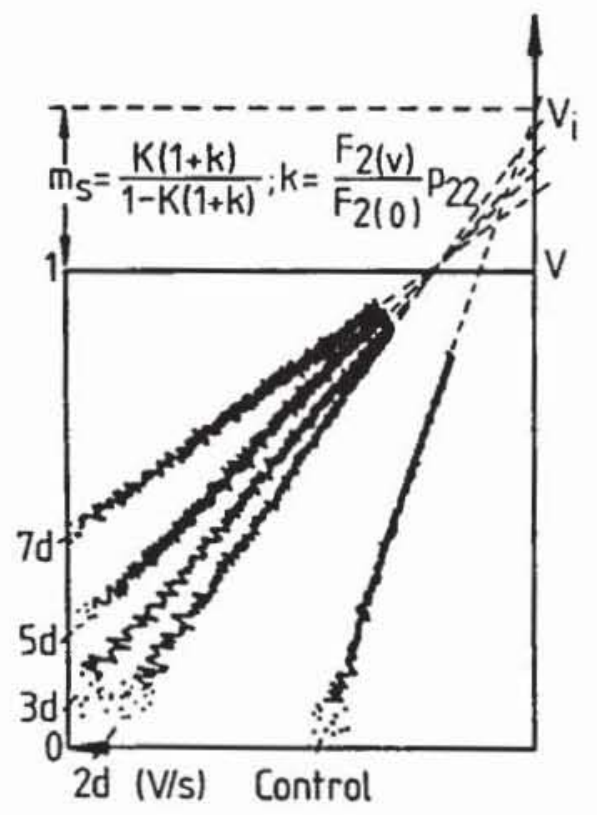

Fig. 2. Calculations of the grouping probabilities $p_{22}$ from the intersept $V$, of the $V$ versus $V / s$ plots during a $7 \mathrm{~d}$ treatment with $160 \mu$ M BAS $13-338$ (SAN 9785). $K=0.145$.
( $k=0$, the slope of the plot is $m_{3}=K /(1-K)$ ) $K$, the temperature constant for charge transfer of a reduced reaction center to oxidized internal acceptors (pheophytin, $\mathrm{Q}_{\mathrm{A}}$ ) can be calculated. $K$ has the value $0.145[16]$ which is valid for most higher plants. The effect of an altered lipid matrix on energy distribution is shown in Fig. 3. As presented in Table II, nearly all energy distribution parameters are affected following treatment with BAS 13-338 (SAN 9785). As can be shown by apparent quantum yields $\varphi_{1 j}$ of an individual photosystem and by energy transfer probabilities $p_{\|}$, trapping as well as grouping are decreasing, whereas spillover and dissipation are increasing during the pyridazinone treatment. However, it has to be emphasized that grouping is almost zero whereas the energy transfer probability $p_{2 b}$ for PS II trapping seems to be unaffected by an altered lipid matrix. Furthermore, decreases of the conformation constants $k_{1}$ for trapping, grouping and spillover as well as increases in the relative distances $R_{\text {II }}$ between the different loci might reflect functional changes and/or a rearrangement of pigment protein complexes caused by structural changes in the lipid environment.

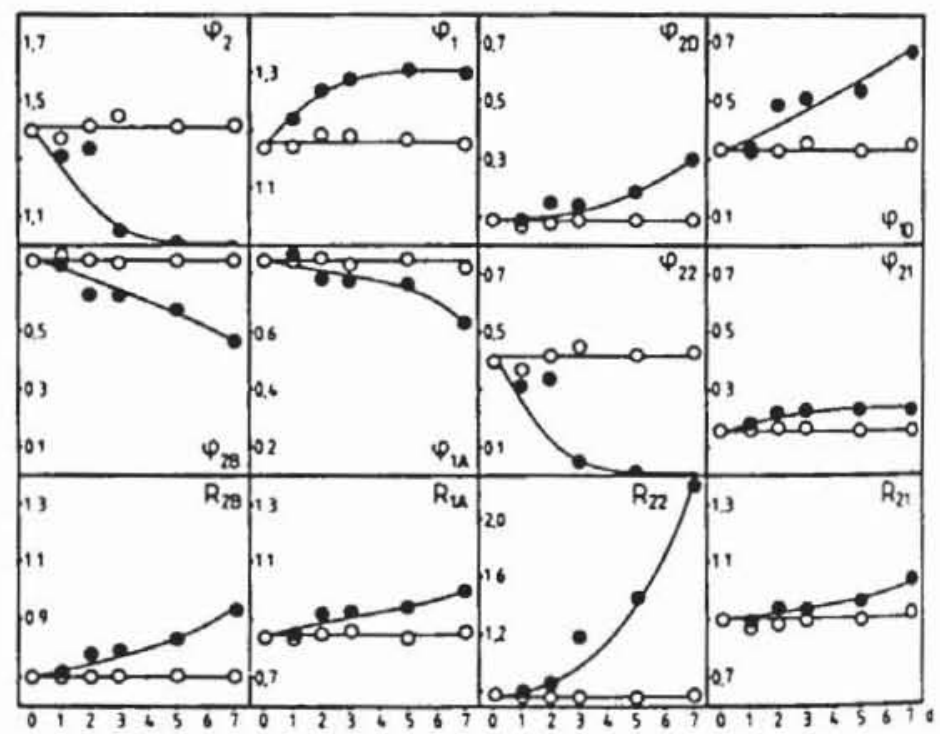

Fig. 3. Energy distribution terms $\varphi_{1}$ and relative distances $R_{11}$, where $\varphi_{11}$ gives the quantum yield of an energy flux $E_{11}$ ( $E_{1 /}$ /absorption of PS II or PS I). $R_{11}$ represents the relative distances between the pigment protein complexes $i$ and $j$ calculated from the conformation constants using the FORSTER-resonance-relationship: $k_{11}^{-1}=R_{11}^{6}$. O Control: - $160 \mu \mathrm{M}$ BAS 13-338 (SAN 9785). 
Table II. Energy distribution terms following $7 \mathrm{~d}$ treatment with $160 \mu \mathrm{M}$ BAS 13-338 (SAN 9785). Relative values are mean $\pm S D$ from at least 4 experiments.

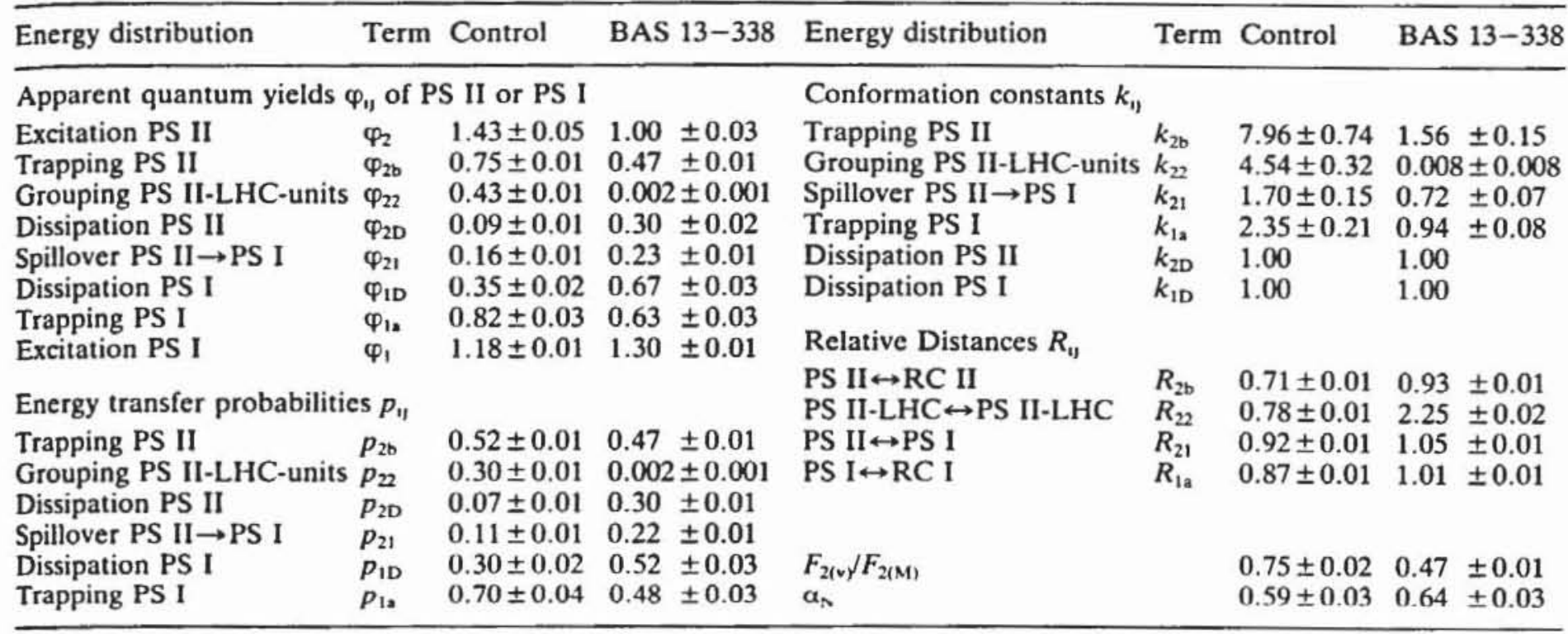

\section{Discussion}

It has been proposed that the shape of a given acyl lipid molecule is mainly determined by the ratio of polar head group and the type of acyl moieties [17]. Therefore, lipid packing properties are expected to bring about asymmetrical distribution of lipid classes and lipid species in membranes. The pyridazinone BAS 13-338 (SAN 9785) induces alterations of the ultrastructure of thylakoid membranes by increasing the ratio of appressed/nonappressed regions in grana [10]. This effect may be attributed to changes in fatty acid composition from a more unsaturated (high linolenic acid content) to a more saturated (low linolenic acid content) state of the thylakoid lipid matrix. Changes in the unsaturation of fatty acids are expected to cause changes in lipid shapes provided that the acyl lipid composition is constant. Such changes may be e.g. from cone-shaped to cylindricalshaped MGDG. Therefore, remarkable changes in the lipid shape are thought to induce alterations in the organization of the lipid matrix. Our calculations imply that the frequencies of $18: 3 / 18: 3$ species in MGDG, DGDG and SQDG are lowered by nearly $50 \%$. In addition, our results indicate that energy transfer between different pigment protein complexes depends on close distances between them and could be influenced by the state of the lipid matrix. These findings are in agreement with the proposed relationship between pyridazinone induced altera- tions in linolenic acid content and fluorescence induction in vivo [18].

Calculations in [19] propose that in appressed regions, there is a minimum set-up of acyl lipids which favour energy transfer between pigment protein complexes. All our data suggest that the amount of linolenic acid in glycolipids is strongly correlated (significance $0.1-5 \%$ ) with excitation, trapping and dissipation of both PS II and PS I, with grouping of PS II-LHC-units, but not with spillover. From these findings we conclude that a relationship exists between the structural organization of the lipid matrix and the functional state of energy distribution in thylakoids.

Considering the possible mechanism of the BAS 13-338 (SAN 9785) effect on energy distribution, we assume that due to pyridazinone induced alterations in shape, glycolipids are inserted into appressed rather than into non-appressed regions of thylakoids by lipid turnover metabolism. This might result in an altered lipid micro-environment as well as in a rearrangement of pigment protein complexes (increased distances between these complexes) in appressed regions. Structural changes in the organization of the lipid matrix are expected to cause changes in the functional energy distribution behaviour by creating a new state of lipid-protein interaction.

On the basis of our data, possible models for the effect of the pyridazinone BAS 13-338 (SAN 9785) 
LEECH et al. 1985

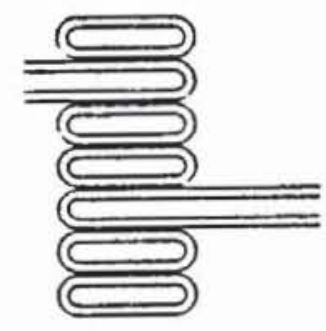

LEECH et al. 1985

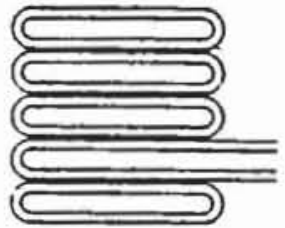

Thylakoids proposed: GRAF et al. 1986
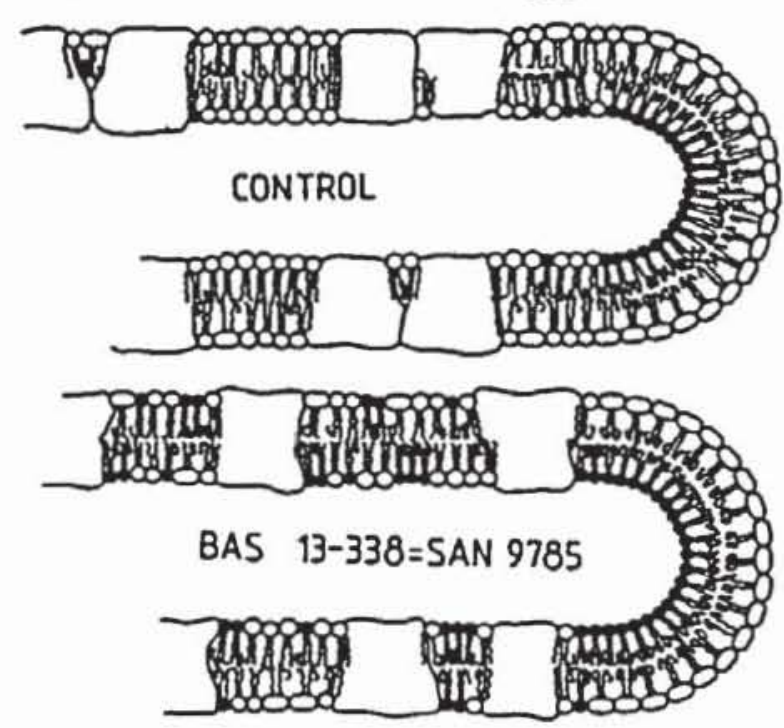
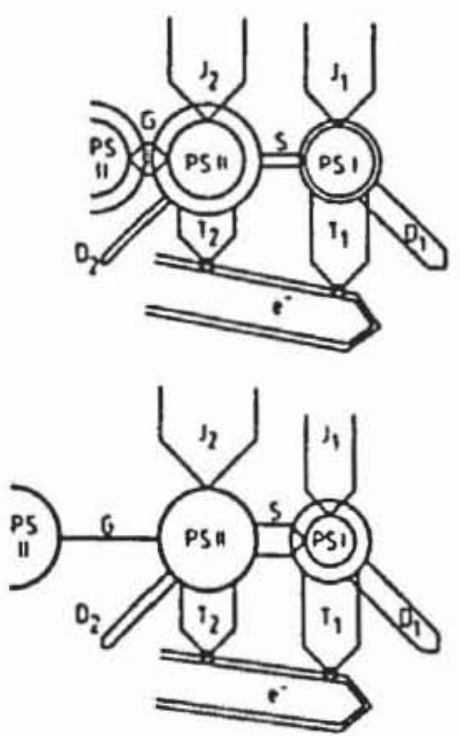

Fig. 4. Grana models: according to Leech et al. (1985). Thylakoid models: the models show a thylakoid sac originating from different appressed regions of grana stacks, respectively. Energy distribution models: data calculated from $77 \mathrm{~K}$ fluorescence kinetics are compiled in a synergetic way visualizing energy distribution constellation in thylakoids after $7 \mathrm{~d}$ treatment with $160 \mu \mathrm{M}$ BAS 13-338. $\mathrm{J}=$ absorption, $\mathrm{T}=$ trapping. $\mathrm{G}=$ grouping. $\mathrm{S}=$ spillover, $\mathrm{D}=$ dissipation, $\mathrm{e}^{-}=$electron transport chain.

on thylakoids are proposed (Fig. 4). In these thylakoid models, the structure of the lipid matrix is related to the functional state of energy distribution in a grouped bipartite model. Both models are in agreement with the observed pyridazinone induced changes in grana size [10].

[1] B. Andersson and J. M. Anderson, Biochim. Biophys. Acta 593, 427-440 (1980).

[2] R. J. Strasser. Ber. Deutsch. Bot. Ges. 98, 53-72 (1985).

[3] W. P. Williams, K. Gounaris, and P. J. Quinn, in: Advances in Photosynthesis Research III (C. Sybesma, ed.), p. 123-130. Nijhoff/Junk Publishers. The Hague 1984.

[4] A. Radunz, K. P. Bader, and G. H. Schmid. Z. Pflanzenphysiol. 114, 227-231 (1984).

[5] D. J. Chapman. J. De-Felice, and J. Barber, Planta 166, 280-285 (1985).

[6] J. A. Graf, R. J. Strasser, and U. Kull, in: Advances in Photosynthesis Research IV (C. Sybesma, ed.), p. 37-40, Nijhoff/Junk Publishers. The Hague 1984.

[7] R. M. Mannan and S. Bose, Photochem. Photobiol. 41, 63-72 (1985).

[8] J. B. St. John and M. N. Christiansen. Plant Physiol. 57, 257-259 (1976).

[9] N. W. Lem and J. P. Williams, Plant Physiol. 68, 944-949 (1981).

[10] R. M. Leech, C. A. Walton, and N. R. Baker. Planta $165,277-283$ (1985).

\section{Acknowledgements}

We are grateful to BASF AG, Limburgerhof, and SANDOZ AG, Basel, for the supply of the pyridazinone used in this study.

[11] E. Weis, Biochim. Biophys. Acta 807, 118-126 (1985).

[12] R. J. Strasser, in: Chloroplast Development (G. Akoyunoglou and J. H. Argyroudi-Akoyunoglou. eds.). p. 513-524, Elsevier, Amsterdam 1978.

[13] E. G. Bligh and W. J. Dyer, Can. J. Biochem. Physiol. 37, $911-917$ (1959).

[14] U. Kull and K. Jeremias. Z. Pflanzenphysiol. 68, 55-62 (1972).

[15] W. L. Butler, Ann. Rev. Plant Physiol. 29, 345-378 (1978).

[16] J. A. Graf, R. J. Strasser, and U. Kull, in: Proceedings VIIth International Congress on Photosynthesis III (J. Biggins, ed.). pp. 713-716. Nijhoff/Junk Publishers, The Hague 1986.

[17] D. J. Murphy, FEBS Lett. 150, 19-26 (1983).

[18] G. Laskay, E. Lehoczki. A. L. Dobi, and L. Szalay, Planta 169, 123-129 (1986)

[19] D. J. Murphy, Biochim. Biophys. Acta 864, 33-94 (1986). 